\title{
A note on vertices of indecomposable tensor products
}

\author{
Markus Linckelmann \\ Communicated by Christopher W. Parker
}

\begin{abstract}
G. Navarro raised the question of when two vertices of two indecomposable modules over a finite group algebra generate a Sylow $p$-subgroup. The present note provides a sufficient criterion for this to happen. This generalises a result by Navarro for simple modules over finite $p$-solvable groups, which is the main motivation for this note.
\end{abstract}

Let $p$ be a prime and $\mathcal{O}$ a complete local principal ideal domain with residue field $k$ of characteristic $p$. We allow the case $\mathcal{O}=k$, unless stated otherwise. We assume that $k$ is large enough for the modules and their sources that appear in this note to be absolutely indecomposable; the point of this hypothesis is that it ensures that indecomposable modules over finite group algebras have multiplicity modules (see $[9, \S 5.7]$ for background material). If the field of fractions $K$ of $\mathcal{O}$ has characteristic zero, then we also assume that $K$ is large enough so that the irreducible characters over $K$ that arise below are absolutely irreducible. In some of the results in the literature cited below, $k$ is algebraically closed for convenience, but it is easy to see that "large enough" in the sense above will do for the quoted results. Modules are finitely generated left modules.

Theorem 1. Let $G$ be a finite group, and let $U, V$ be indecomposable $\mathcal{O}$-free $\mathcal{O} G$ modules having sources of $\mathcal{O}$-ranks prime to $p$. Suppose that $W=U \otimes_{\mathcal{O}} V$ is indecomposable and that $W$ has a simple multiplicity module. Then the sources of $W$ have $\mathcal{O}$-rank prime to $p$, and there exist vertices $Q, R$ of $U, V$, respectively, such that $Q \cap R$ is a vertex of $W$ and such that $Q R$ is a Sylow p-subgroup of $G$.

The proof of Theorem 1 yields some further technical information regarding which intersections of vertices of $U$ and $V$ yield vertices of $W$ (cf. Remark 9), and some information regarding the $p$-parts of the ranks of $U, V, W$ and the dimensions of their multiplicity modules (cf. Remark 10). The proof shows also that the hypothesis on the simplicity of the multiplicity module of $W$ can be replaced by a slightly weaker condition on the dimension of the multiplicity module of $W$ (cf. Remark 11). See Külshammer [8, Proposition 2.1] for a sufficient criterion for when the tensor product of two modules is indecomposable. We note some 
immediate consequences of Theorem 1. By a result of Knörr (in the proof of [7, Proposition 3.1], also described in [9, Corollary 5.7.9]), simple $k G$-modules and $\mathcal{O} G$-lattices with irreducible characters have simple multiplicity modules. Thus Theorem 1 implies the following two results.

Corollary 2. Let $G$ be a finite group, and let $U, V$ be simple $k G$-modules with sources of dimensions prime to $p$ such that $W=U \otimes_{k} V$ is simple. Then the sources of $W$ have dimension prime to $p$, and there exist vertices $Q, R$ of $U, V$, respectively, such that $Q \cap R$ is a vertex of $W$ and such that $Q R$ is a Sylow p-subgroup of $G$.

Corollary 3. Suppose that the field of fractions $K$ of $\mathcal{O}$ has characteristic zero. Let $U, V$ be $\mathcal{O}$-free $\mathcal{O} G$-modules with irreducible characters and sources of $\mathcal{O}$-ranks prime to $p$. Suppose that the character of $W=U \otimes_{\mathcal{O}} V$ is irreducible. Then the sources of $W$ have $\mathcal{O}$-rank prime to $p$, and there exist vertices $Q, R$ of $U, V$, respectively, such that $Q \cap R$ is a vertex of $W$ and such that $Q R$ is a Sylow $p$-subgroup of $G$.

By a result in unpublished notes of Puig from 1988 (see e.g. [16, Theorem (30.5)] or [9, Theorem 10.6.8], also attributed to Feit in [14]), if $G$ is a finite $p$-solvable group, then simple $k G$-modules have endopermutation sources; in particular, their sources have dimensions prime to $p$. Thus Corollary 2 implies the following result due to Navarro (and this is the main motivation for this note).

Corollary 4 (Navarro [13, Theorem A]). Let $G$ be a finite p-solvable group, and let $U, V$ be simple $k G$-modules such that $W=U \otimes_{k} V$ is simple. Then there exist vertices $Q, R$ of $U, V$, respectively, such that $Q \cap R$ is a vertex of $W$ and such that $Q R$ is a Sylow p-subgroup of $G$.

For the proof of Theorem 1, we collect a few elementary observations on vertices and sources which imply that the first two statements in Theorem 1 hold under weaker hypotheses. We refer to $[9, \S 5.1]$ for definitions and basic properties of Green's theory of vertices and sources of modules from [6]; these - as well as a number of arguments in this note - depend on the fact, used without further mention, that the Krull-Schmidt theorem holds in the context of the present note. The following is well known.

Lemma 5 (see e.g. [9, Theorem 5.1.11] or [12, Chapter 3, Theorem 1.17]). Let $G$ be a finite group, and let $U, V$ be indecomposable $\mathcal{O}$-free $\mathcal{O} G$-modules, and let $Q, R$ be vertices of $U, V$, respectively. Then, for every indecomposable direct summand $W$ of $U \otimes_{\mathcal{O}} V$, there is $x \in G$ such that $Q \cap{ }^{x} R$ contains a vertex of $W$. 
Lemma 6. Let $G$ be a finite group, and let $U, V$ be indecomposable $\mathcal{O}$-free $\mathcal{O} G$ modules having sources of $\mathcal{O}$-ranks prime to $p$.

(i) For any $x \in G$, there exists an indecomposable direct summand

$$
W \text { of } U \otimes_{\mathcal{O}} V
$$

such that $Q \cap{ }^{x} R$ is contained in a vertex of $W$.

(ii) If $x \in G$ is chosen such that $Q \cap{ }^{x} R$ has maximal order amongst the subgroups of the form $Q \cap{ }^{y} R$, where $y \in G$, then $U \otimes_{\mathcal{O}} V$ has an indecomposable direct summand $W$ with vertex $Q \cap{ }^{x} R$ and with sources of $\mathcal{O}$-rank prime to $p$.

(iii) Suppose that $U \otimes_{\mathcal{O}} V$ is indecomposable. Then the sources of $U \otimes_{\mathcal{O}} V$ have $\mathcal{O}$-rank prime to $p$, and for any $x \in G$, the subgroup $Q \cap{ }^{x} R$ is contained in a vertex of $U \otimes_{\mathcal{O}} V$. If $Q \cap{ }^{x} R$ has maximal order amongst all subgroups of this form, then $Q \cap{ }^{x} R$ is a vertex of $U \otimes_{\mathcal{O}} V$.

Proof. Let $Q, R$ be vertices of $U, V$, respectively. Let $x \in G$. Then $Q \cap{ }^{x} R$ is contained in a vertex of $U$ and in a vertex of $V$. The assumptions on $U$ and $V$ imply that $\operatorname{Res}_{Q \cap x}^{G}(U)$ and $\operatorname{Res}_{Q \cap{ }^{x} R}^{G}(V)$ have indecomposable direct summands of $\mathcal{O}$-ranks prime to $p$. Thus their tensor product $\operatorname{Res}_{Q \cap^{x}{ }_{R}}^{G}\left(U \otimes_{\mathcal{O}} V\right)$ has an indecomposable direct summand $Y$ of $\mathcal{O}$-rank prime to $p$. In particular, $Q \cap{ }^{x} R$ is the vertex of $Y$. Moreover, since $Y$ is indecomposable, it follows that $Y$ is isomorphic to a direct summand of $\operatorname{Res}_{Q \cap{ }^{x} R}^{G}(W)$ for some indecomposable direct summand $W$ of $U \otimes_{\mathcal{O}} V$, and hence $Q \cap{ }^{x} R$ is contained in a vertex of $W$. This shows (i). The same argument, assuming in addition that $Q \cap{ }^{x} R$ has maximal order amongst the subgroups of this form, in conjunction with Lemma 5 shows (ii). Statement (iii) follows from (i) and (ii).

For $m$ a positive integer, we denote by $m_{p}$ the highest power of $p$ which divides $m$, and we denote by $\operatorname{rk}_{\mathcal{O}}(U)$ the $\mathcal{O}$-rank of a free $\mathcal{O}$-module $U$.

Lemma 7 (Green [6, Theorem 9]). Let $G$ be a finite group, $U$ an $\mathcal{O}$-free indecomposable $\mathcal{O} G$-module, and $Q$ a vertex of $U$. Then $\operatorname{rk}_{\mathcal{O}}(U)_{p} \geq|G: Q|_{p}$.

See e.g. [9, Theorem 5.12.13] or [12, Chapter 7, Theorem 7.5] for proofs of this lemma. The key ingredient for the proof of Theorem 1 is the following result due to Knörr, which is a criterion for when the inequality in Lemma 7 is an equality.

Theorem 8 ([7, Theorem 4.5]). Let $G$ be a finite group, $U$ an indecomposable $\mathcal{O}$-free $\mathcal{O} G$-module, and $Q$ a vertex of $U$. Suppose that the sources of $U$ have $\mathcal{O}$-rank prime to $p$ and that $U$ has a simple multiplicity module. Then

$$
\operatorname{rk}_{\mathcal{O}}(U)_{p}=|G: Q|_{p} .
$$


The statement in [7, Theorem 4.5] does not mention multiplicity modules explicitly, but the hypotheses in the theorem ensure the simplicity of multiplicity modules, and this is all that is used in the proof of [7, Theorem 4.5]. See [15, §9] for more general background material on multiplicity modules and characterisations of simple multiplicity modules. A description of some of this material closer to the terminology used above is given in [9, Theorems 5.7.7, 5.12.15].

Proof of Theorem 1. Let $Q, R$ be vertices of $U, V$, respectively, chosen such that $Q \cap R$ has maximal order amongst all intersections of a vertex of $U$ and a vertex of $V$. It follows from Lemma 6 (iii) that $Q \cap R$ is a vertex of $W=U \otimes_{\mathcal{O}} V$ and that the sources of $W$ have $\mathcal{O}$-rank prime to $p$.

Since $W$ has a simple multiplicity module, it follows from Theorem 8 and Lemma 7 that we have

$$
\begin{aligned}
|G: Q \cap R|_{p} & =\operatorname{rk}_{\mathcal{O}}(W)_{p}=\operatorname{rk}_{\mathcal{O}}(U)_{p} \cdot \operatorname{rk}_{\mathcal{O}}(V)_{p} \\
& \geq|G: Q|_{p} \cdot|G: R|_{p} .
\end{aligned}
$$

The left side is also equal to $|G: Q|_{p} \cdot|Q: Q \cap R|$; hence cancelling $|G: Q|_{p}$ yields

$$
|Q: Q \cap R| \geq|G: R|_{p}=|G|_{p} /|R| .
$$

Now $|Q: Q \cap R|=|Q R| /|R|$, so together this yields $|Q R| \geq|G|_{p}$.

Let $P$ be a Sylow $p$-subgroup of $G$ containing $Q$. Let $x \in G$ such that ${ }^{x} R \subseteq P$. It follows from Lemma 6 (ii) that $Q \cap{ }^{x} R$ is contained in a vertex of $W$. In particular, we have $\left|Q \cap{ }^{x} R\right| \leq|Q \cap R|$. Thus $\left|Q\left({ }^{x} R\right)\right| \geq|Q R| \geq|P|$. But since both $Q,{ }^{x} R$ are contained in $P$, we also have $\left|Q\left({ }^{x} R\right)\right| \leq|P|$. Thus all inequalities in this proof are equalities. This forces $Q\left({ }^{x} R\right)=P$ and $\left|Q \cap{ }^{x} R\right|=|Q \cap R|$, so $Q$ and ${ }^{x} R$ (instead of $R$ ) satisfy all conclusions.

Remark 9. The proof of Theorem 1 shows a bit more: with the notation and hypotheses of Theorem 1, any intersection of a vertex of $U$ and a vertex of $V$ is contained in a vertex of $W$, and any intersection of maximal order of a vertex of $U$ and a vertex of $V$ is a vertex of $W$. Moreover, for any choice of vertices $Q, R$ of $U, V$, respectively, such that both $Q, R$ are contained in a Sylow $p$-subgroup $P$ of $G$, we have $P=Q R$, and $Q \cap R$ is a vertex of $W$. This points to some information about fusion in $G$ : if $x \in G$ is chosen such that $Q \cap{ }^{x} R$ has maximal order amongst all subgroups of this form, then $Q \cap{ }^{x} R$ is a vertex of $W$, and by Lemma 6 (iii), for any $y \in G$, the group $Q \cap^{y} R$ is $G$-conjugate to a subgroup of $Q \cap{ }^{x} R$. In particular, if $x, y$ are two elements in $G$ such that $Q \cap{ }^{x} R$ and $Q \cap{ }^{y} R$ both have the same maximal order amongst all groups of this form, then $Q \cap{ }^{x} R$ and $Q \cap{ }^{y} R$ are $G$-conjugate since they both are vertices of $W$. 
Remark 10. With the notation and hypotheses of Theorem 1 , denote by $(Q, X)$, $(R, Y),(S, Z)$ vertex-source pairs of $U, V, W$, respectively. The fact that the displayed inequalities in the proof of Theorem 1 are all equalities implies that we have

$$
\begin{aligned}
|G: Q|_{p} & =\operatorname{rk}_{\mathcal{O}}(U)_{p}, \\
|G: R|_{p} & =\operatorname{rk}_{\mathcal{O}}(V)_{p}, \\
|G: S|_{p} & =\operatorname{rk}_{\mathcal{O}}(W)_{p} .
\end{aligned}
$$

Moreover, by [9, Theorem 5.12.15], the associated multiplicity modules $M_{U}, M_{V}$, $M_{W}$ of $U, V, W$, respectively, satisfy

$$
\begin{aligned}
\operatorname{dim}_{k}\left(M_{U}\right)_{p} & =\left|N_{G}(Q, X) / Q\right|_{p}, \\
\operatorname{dim}_{k}\left(M_{V}\right)_{p} & =\left|N_{G}(R, Y) / R\right|_{p}, \\
\operatorname{dim}_{k}\left(M_{W}\right)_{p} & =\left|N_{G}(S, Z) / S\right|_{p} .
\end{aligned}
$$

Remark 11. Theorem 1 holds with slightly weaker hypotheses on the multiplicity modules of $W$. Instead of requiring the simplicity of a multiplicity module of $W$, it suffices to require the equality

$$
\operatorname{dim}_{k}\left(M_{W}\right)_{p}=\left|N_{G}(S, Z) / S\right|_{p}
$$

for the multiplicity module $M_{W}$ of $W$ associated with a vertex-source pair $(S, Z)$ of $W$ (this is the last equality in Remark 10). By a result of Knörr [7, Proposition 4.2], this equality holds if $M_{W}$ is simple (essentially because $M_{W}$ is then a projective simple module of a finite central $p^{\prime}$-extension of $N_{G}(S, Z) / S$ ), but the simplicity of $M_{W}$ is not necessary in general for this equality. In particular, even if $M_{W}$ is simple, we do not know whether this implies that $M_{U}, M_{V}$ are necessarily simple as well.

Remark 12. With the notation and hypotheses of Theorem 1, if $D, E$ are defect groups of the blocks to which $U, V$ belong, respectively, such that $D, E$ are both contained in a fixed Sylow $p$-subgroup $P$ of $G$, then $D$ and $E$ contain vertices of $U$ and $V$, respectively, and hence $P=D E$ by Remark 9. It would be interesting to investigate whether there are more precise relationships between the defect groups of the three blocks to which $U, V, W$ belong. For the remainder of this Remark, suppose that all three modules $U, V, W$ have simple multiplicity modules (this is for instance the case in any of the Corollaries $2,3,4)$. Then, as a consequence of the results of Knörr in [7], the vertices $Q, R, Q \cap R$ of $U, V, W$ in Theorem 1 are centric in the fusion systems of the blocks to which these modules belong (see [10, Theorem 10.3.1] for a formulation of [7, Theorem 3.3] in terms of fusion systems of blocks). Thus, for instance, if the block of $\mathcal{O} G$ to which $U$ (resp. $V, W$ ) 
belongs has abelian defect groups, then $Q$ (resp. $R, Q \cap R$ ) is a defect groups of this block. Furthermore, by a slight generalisation [10, Theorem 10.3.6] of a result of Erdmann [5], if $Q$ (resp. $R, Q \cap R$ ) in Theorem 1 is cyclic, then it is a defect group of the block to which $U$ (resp. $V, W$ ) belongs.

Remark 13. E. Giannelli pointed out that the situation is well understood for simple tensor products of simple modules over symmetric groups. Let $n$ be a positive integer. Let $U, V$ be simple $k S_{n}$-modules of dimensions greater than 1 such that $W=U \otimes_{k} V$ is simple. Then, by [2, Main Theorem], we have $p=2$ and $n$ is even. The exact tensor products which can arise in this way are described in [11], proving a conjecture of Gow and Kleshchev. It follows from that description that then $n=2 m$ for some odd integer $m$ and that $U$ can be chosen to be the basic spin module (labelled by the partition $(m+1, m-1)$ ). By [4, Theorem 1.1], the vertices of $U$ are the Sylow 2-subgroups of $S_{n}$. It is, however, not always possible to choose vertices $Q, R$ of $U, V$, respectively, such that $Q R$ is a Sylow 2-subgroup and such that $Q \cap R$ is a vertex of $W$. Note that $U$ has sources of even dimension (cf. [1, Lemma 5.3] and [4, Theorem 6.1]), so the hypotheses of Theorem 1 are not satisfied. The following example is due to E. Giannelli. Consider the case $n=6$, with simple $k S_{6}$-modules $U, V, W$ labelled by the partitions $(4,2),(5,1),(3,2,1)$, respectively. It follows from [11, Theorem 1.1] that we have $W \cong U \otimes_{k} V$. As mentioned above, [4, Theorem 1.1] implies that $U$ has a Sylow 2-subgroup $Q$ of $S_{6}$ as a vertex. Moreover, [3, Theorem 1.2] implies that also $V$ has a Sylow 2-subgroups $R$ of $S_{6}$ as a vertex. The product $Q R$ is therefore a Sylow 2-subgroup of $S_{6}$ if and only if $Q=R$. In that case, we have $Q \cap R=Q=R$, but $W$ has a trivial vertex. In general, for $G$ an arbitrary finite group and $U, V, W$ simple $k G$-modules such that $W \cong U \otimes_{k} V$, it seems to be unknown at present whether there is always a choice of vertices $Q, R$ of $U, V$, respectively, such that $Q$ and $R$ generate a Sylow $p$-subgroup of $G$.

\section{Bibliography}

[1] D. Benson, Spin modules for symmetric groups, J. Lond. Math. Soc. (2) 38 (1988), no. 2, 250-262.

[2] C. Bessenrodt and A. S. Kleshchev, On tensor products of modular representations of symmetric groups, Bull. Lond. Math. Soc. 32 (2000), no. 3, 292-296.

[3] S. Danz and E. Giannelli, Vertices of simple modules of symmetric groups labelled by hook partitions, J. Group Theory 18 (2015), no. 2, 313-334.

[4] S. Danz and B. Külshammer, The vertices and sources of the basic spin module for the symmetric group in characteristic 2, J. Pure Appl. Algebra 213 (2009), no. 7, $1264-1282$. 
[5] K. Erdmann, Blocks and simple modules with cyclic vertices, Bull. Lond. Math. Soc. 9 (1977), no. 2, 216-218.

[6] J. A. Green, On the indecomposable representations of a finite group, Math. Z. 70 (1958/59), 430-445.

[7] R. Knörr, On the vertices of irreducible modules, Ann. of Math. (2) 110 (1979), no. 3 , 487-499.

[8] B. Külshammer, Some indecomposable modules and their vertices, J. Pure Appl. Algebra 86 (1993), no. 1, 65-73.

[9] M. Linckelmann, The Block Theory of Finite Group Algebras. Vol. I, London Math. Soc. Stud. Texts 91, Cambridge University, Cambridge, 2018.

[10] M. Linckelmann, The Block Theory of Finite Group Algebras. Vol. II, London Math. Soc. Stud. Texts 92, Cambridge University, Cambridge, 2018.

[11] L. Morotti, Irreducible tensor products for symmetric groups in characteristic 2, Proc. Lond. Math. Soc. (3) 116 (2018), no. 6, 1553-1598.

[12] H. Nagao and Y. Tsushima, Representations of Finite Groups, Academic Press, Boston, 1989.

[13] G. Navarro, Products of characters, product of subgroups, Notes (2019).

[14] P. Schmid, Endo-permutation modules in p-solvable groups, J. Algebra 129 (1990), no. $1,75-95$.

[15] J. Thévenaz, Duality in G-algebras, Math. Z. 200 (1988), no. 1, 47-85.

[16] J. Thévenaz, G-algebras and Modular Representation Theory, Oxford Math. Monogr., Oxford University, New York, 1995.

Received September 4, 2019; revised October 29, 2019.

\section{Author information}

Markus Linckelmann, Department of Mathematics, City, University of London,

London EC1V 0HB, United Kingdom.

E-mail: markus.linckelmann.10city.ac.uk 